\title{
Photophysical and Structural Properties of Cyanoruthenate Complexes of Hexaaza-triphenylene
}

\author{
Juan-Manuel Herrera, Simon J. A. Pope, Anthony J. H. M. Meijer, \\ Timothy L. Easun, Harry Adams, Wassim Z. Alsindi, Xue-Zhong Sun, \\ Michael W. George, Stephen Faulkner and Michael D. Ward*
}

Supporting information

Complete reference 38:

(38) Gaussian 03, Revision B.05: Frisch, M. J.; Trucks, G. W.; Schlegel, H. B.; Scuseria, G. E.; Robb, M. A.; Cheeseman, J. R.; Montgomery, Jr., J. A.; Vreven, T.; Kudin, K. N.; Burant, J. C.; Millam, J. M.; Iyengar, S. S.; Tomasi, J.; Barone, V.; Mennucci, B.; Cossi, M.; Scalmani, G.; Rega, N.; Petersson, G. A.; Nakatsuji, H.; Hada, M.; Ehara, M.; Toyota, K.; Fukuda, R.; Hasegawa, J.; Ishida, M.; Nakajima, T.; Honda, Y.; Kitao, O.; Nakai, H.; Klene, M.; Li, X.; Knox, J. E.; Hratchian, H. P.; Cross, J. B.; Bakken, V.; Adamo, C.; Jaramillo, J.; Gomperts, R.; Stratmann, R. E.; Yazyev, O.; Austin, A. J.; Cammi, R.; Pomelli, C.; Ochterski, J. W.; Ayala, P. Y.; Morokuma, K.; Voth, G. A.; Salvador, P.; Dannenberg, J. J.; Zakrzewski, V. G.; Dapprich, S.; Daniels, A. D.; Strain, M. C.; Farkas, O.; Malick, D. K.; Rabuck, A. D.; Raghavachari, K.; Foresman, J. B.; Ortiz, J. V.; Cui, Q.; Baboul, A. G.; Clifford, S.; Cioslowski, J.; Stefanov, B. B.; Liu, G.; Liashenko, A.; Piskorz, P.; Komaromi, I.; Martin, R. L.; Fox, D. J.; Keith, T.; AlLaham, M. A.; Peng, C. Y.; Nanayakkara, A.; Challacombe, M.; Gill, P. M. W.; Johnson, B.; Chen, W.; Wong, M. W.; Gonzalez, C.; and Pople, J. A.; Gaussian, Inc., Wallingford CT, 2004.

Table S1. First 10 excitation wavelengths (in $\mathrm{nm}$ ) for each complex in water and DMF, calculated using TD-DFT. See text for additional details.

\begin{tabular}{|c|c|c|c|c|c|c|}
\hline \# & {$[1]^{2-}$ (DMF) } & {$[2]^{4-}$ (DMF) } & {$[3]^{6-}(\mathrm{DMF})$} & {$[1]^{2-} \cdot 4 \mathrm{H}_{2} \mathrm{O}$} & {$[2]^{4-} \cdot 8 \mathrm{H}_{2} \mathrm{O}$} & {$[3]^{6-\bullet} \cdot 12 \mathrm{H}_{2} \mathrm{O}$} \\
\hline 1 & 737.76 & 761.85 & 792.06 & 664.00 & 700.43 & 741.24 \\
\hline 2 & 711.89 & 761.55 & 790.23 & 642.07 & 694.75 & 738.92 \\
\hline 3 & 645.78 & 721.61 & 790.02 & 585.90 & 664.98 & 738.33 \\
\hline 4 & 623.86 & 720.46 & 752.26 & 563.13 & 664.63 & 706.97 \\
\hline 5 & 580.09 & 697.80 & 728.05 & 530.25 & 644.03 & 685.34 \\
\hline 6 & 555.70 & 664.81 & 727.96 & 506.86 & 616.22 & 685.21 \\
\hline 7 & 545.75 & 652.72 & 727.72 & 504.30 & 603.11 & 684.93 \\
\hline 8 & 539.34 & 644.59 & 703.67 & 488.64 & 593.80 & 666.91 \\
\hline 9 & 494.24 & 642.88 & 703.66 & 464.48 & 593.76 & 666.63 \\
\hline 10 & 363.79 & 596.63 & 701.11 & 364.72 & 558.57 & 662.94 \\
\hline
\end{tabular}


Table S2. Coordinates $(\AA)$ of energy-minimised structures used for DFT calculations.

Coordinates of $[3]^{6-}$ in $\mathrm{dmf}$

$\begin{array}{cccc}\text { Atom } & \mathrm{X} & \mathrm{Y} & \mathrm{Z} \\ \mathrm{C} & -0.000203 & -0.002622 & -0.000065 \\ \mathrm{C} & -0.000138 & -0.003103 & 1.434513 \\ \mathrm{C} & 1.216180 & -0.002092 & -0.702639 \\ \mathrm{C} & 1.215873 & -0.001577 & 2.137291 \\ \mathrm{C} & 2.458415 & 0.000799 & 1.419404 \\ \mathrm{C} & 2.458492 & 0.000101 & 0.015211 \\ \mathrm{C} & 0.061450 & -0.002659 & 4.141620 \\ \mathrm{C} & -1.160165 & -0.004084 & 3.435701 \\ \mathrm{H} & -2.101399 & -0.006097 & 3.971960 \\ \mathrm{H} & 0.066969 & -0.003638 & 5.224866 \\ \mathrm{C} & -1.159526 & -0.002683 & -2.001555 \\ \mathrm{C} & 0.062167 & -0.002720 & -2.707093 \\ \mathrm{H} & -2.100603 & -0.003658 & -2.538069 \\ \mathrm{H} & 0.067894 & -0.003120 & -3.790337 \\ \mathrm{C} & 4.771782 & 0.005804 & 1.422762 \\ \mathrm{H} & 5.706708 & 0.008194 & 1.969774 \\ \mathrm{C} & 4.771802 & 0.003942 & 0.011941 \\ \mathrm{H} & 5.706751 & 0.004355 & -0.535029 \\ \mathrm{~N} & -1.216560 & -0.003534 & 2.076890 \\ \mathrm{~N} & -1.216424 & -0.002465 & -0.642648 \\ \mathrm{~N} & 1.267702 & -0.002281 & -2.077221 \\ \mathrm{~N} & 3.623279 & 0.001742 & -0.716458 \\ \mathrm{~N} & 3.623148 & 0.003964 & 2.151100 \\ \mathrm{~N} & 1.267117 & -0.000734 & 3.511831 \\ \mathrm{Ru} & 3.264235 & -0.000955 & -2.812756 \\ \mathrm{Ru} & 3.263601 & 0.001711 & 4.247408 \\ \mathrm{Ru} & -2.852024 & 0.000437 & 0.716446 \\ \mathrm{C} & 3.284909 & 2.048864 & -2.850897 \\ \mathrm{~N} & 3.297499 & 3.247036 & -2.868576 \\ \mathrm{C} & 5.198798 & 0.001935 & -3.292401 \\ \mathrm{~N} & 6.369424 & 0.003565 & -3.553107 \\ \mathrm{C} & 2.713781 & -0.006556 & -4.728038 \\ \mathrm{~N} & 2.355912 & -0.009103 & -5.872705 \\ \mathrm{C} & 3.287205 & -2.050837 & -2.846648 \\ \mathrm{~N} & 3.298080 & -3.248961 & -2.864992 \\ \mathrm{C} & 5.198122 & 0.002406 & 4.727148 \\ \mathrm{~N} & 6.368780 & 0.001797 & 4.987717 \\ \mathrm{C} & 2.712710 & -0.000083 & 6.162535 \\ \mathrm{~N} & 2.354449 & -0.002609 & 7.307077 \\ \mathrm{C} & 3.282825 & 2.051402 & 4.286194 \\ \mathrm{~N} & 3.293149 & 3.249791 & 4.307505 \\ \mathrm{C} & 3.290301 & -2.048287 & 4.278634 \\ \mathrm{~N} & 3.305812 & -3.246481 & 4.291998 \\ \mathrm{C} & -2.902487 & -2.049002 & 0.715936 \\ \mathrm{~N} & -2.931883 & -3.247196 & 0.717593 \\ \mathrm{C} & -4.233931 & 0.004154 & -0.719457 \\ \mathrm{~N} & -5.044909 & 0.006423 & -1.603024 \\ \mathrm{C} & -4.236172 & 0.003296 & 2.150274 \\ \mathrm{~N} & -5.049089 & 0.006307 & 3.032021 \\ \mathrm{C} & -2.884820 & 2.050578 & 0.723961 \\ \mathrm{~N} & -2.897966 & 3.248535 & 0.734130 \\ & & & \end{array}$


Coordinates of $[2]^{4-}$ in $\mathrm{dmf}$

$\begin{array}{cccc}\text { Atom } & \mathrm{X} & \mathrm{Y} & \mathrm{Z} \\ & & & \\ \mathrm{C} & 0.008445 & -0.018652 & -0.004092 \\ \mathrm{C} & 0.021513 & -0.002050 & 1.428336 \\ \mathrm{C} & 1.221068 & -0.015217 & -0.702837 \\ \mathrm{C} & 1.226403 & 0.004787 & 2.181943 \\ \mathrm{C} & 2.502260 & -0.016848 & 1.446775 \\ \mathrm{C} & 2.454480 & -0.021730 & 0.026634 \\ \mathrm{C} & -0.013898 & 0.052005 & 4.128188 \\ \mathrm{C} & -1.227721 & 0.043404 & 3.393350 \\ \mathrm{H} & -2.185548 & 0.057835 & 3.896854 \\ \mathrm{H} & -0.049999 & 0.075496 & 5.216761 \\ \mathrm{C} & -1.152448 & -0.029387 & -2.004541 \\ \mathrm{C} & 0.071951 & -0.023655 & -2.709870 \\ \mathrm{H} & -2.092205 & -0.033101 & -2.541842 \\ \mathrm{H} & 0.078638 & -0.021648 & -3.792311 \\ \mathrm{C} & 4.808679 & -0.043389 & 1.349862 \\ \mathrm{H} & 5.768573 & -0.048165 & 1.864962 \\ \mathrm{C} & 4.781823 & -0.045009 & -0.068812 \\ \mathrm{H} & 5.698537 & -0.047629 & -0.644133 \\ \mathrm{~N} & -1.214947 & 0.014348 & 2.042076 \\ \mathrm{~N} & -1.207559 & -0.029847 & -0.646949 \\ \mathrm{~N} & 1.273901 & -0.011651 & -2.076925 \\ \mathrm{~N} & 3.605515 & -0.038034 & -0.735573 \\ \mathrm{~N} & 3.691952 & -0.027687 & 2.101979 \\ \mathrm{~N} & 1.196932 & 0.030930 & 3.539522 \\ \mathrm{Ru} & 3.246939 & 0.012472 & -2.816796 \\ \mathrm{Ru} & -2.837681 & -0.012318 & 0.687870 \\ \mathrm{C} & 3.257112 & -2.031833 & -2.899131 \\ \mathrm{~N} & 3.244067 & -3.228917 & -2.955782 \\ \mathrm{C} & 5.185658 & 0.026288 & -3.315941 \\ \mathrm{~N} & 6.352311 & 0.032881 & -3.593452 \\ \mathrm{C} & 2.686929 & 0.067358 & -4.727324 \\ \mathrm{~N} & 2.314051 & 0.099837 & -5.866626 \\ \mathrm{C} & 3.250830 & 2.057774 & -2.777316 \\ \mathrm{~N} & 3.227997 & 3.256311 & -2.756071 \\ \mathrm{C} & -2.866509 & 2.032961 & 0.650929 \\ \mathrm{~N} & -2.891448 & 3.230736 & 0.621026 \\ \mathrm{C} & -4.221922 & -0.041247 & -0.747714 \\ \mathrm{~N} & -5.022714 & -0.059085 & -1.640272 \\ \mathrm{~N} & -4.231157 & 0.021571 & 2.116525 \\ \mathrm{~N} & -034914 & 0.042867 & 3.006252 \\ \mathrm{~N} & & -2.057476 & 0.735740 \\ \mathrm{~N} & -3.255284 & 0.762506 \\ \mathrm{~N} & & \end{array}$


Coordinates of $[1]^{2-}$ in $\mathrm{dmf}$

$\begin{array}{cccc}\text { Atom } & \mathrm{X} & \mathrm{Y} & \mathrm{Z} \\ & & & \\ \mathrm{C} & 0.001193 & 0.000047 & -0.009663 \\ \mathrm{C} & -0.002348 & -0.000715 & 1.423467 \\ \mathrm{C} & 1.208981 & 0.001224 & -0.745814 \\ \mathrm{C} & 1.201839 & -0.001454 & 2.165517 \\ \mathrm{C} & 2.470746 & -0.000980 & 1.429547 \\ \mathrm{C} & 2.474285 & 0.001011 & -0.003596 \\ \mathrm{C} & -0.031451 & -0.002776 & 4.122601 \\ \mathrm{C} & -1.242066 & -0.001655 & 3.393659 \\ \mathrm{H} & -2.202082 & -0.001612 & 3.895347 \\ \mathrm{H} & -0.057323 & -0.003816 & 5.210625 \\ \mathrm{C} & -1.228901 & 0.001277 & -1.985881 \\ \mathrm{C} & -0.014771 & 0.002723 & -2.708885 \\ \mathrm{H} & -2.186443 & 0.001303 & -2.492241 \\ \mathrm{H} & -0.035327 & 0.003939 & -3.797020 \\ \mathrm{C} & 4.792777 & -0.000810 & 1.429051 \\ \mathrm{H} & 5.726106 & -0.001913 & 1.988932 \\ \mathrm{C} & 4.796274 & 0.001950 & 0.008429 \\ \mathrm{H} & 5.732354 & 0.003467 & -0.546830 \\ \mathrm{~N} & -1.239594 & -0.000542 & 2.039870 \\ \mathrm{~N} & -1.233000 & -0.000204 & -0.632064 \\ \mathrm{~N} & 1.197850 & 0.002550 & -2.112473 \\ \mathrm{~N} & 3.652605 & 0.002759 & -0.699238 \\ \mathrm{~N} & 3.645627 & -0.002138 & 2.131031 \\ \mathrm{~N} & 1.183996 & -0.002603 & 3.532083 \\ \mathrm{Ru} & -2.864174 & -0.000199 & 0.699637 \\ \mathrm{C} & -2.908597 & 2.049831 & 0.696203 \\ \mathrm{~N} & -2.926296 & 3.247924 & 0.691457 \\ \mathrm{C} & -4.251018 & -0.002144 & -0.737973 \\ \mathrm{~N} & -5.056674 & -0.003543 & -1.626009 \\ \mathrm{C} & -4.258873 & 0.001868 & 2.129808 \\ \mathrm{~N} & -5.069077 & 0.003343 & 3.013679 \\ \mathrm{C} & -2.910631 & -2.050045 & 0.704571 \\ \mathrm{~N} & -2.931812 & -3.248067 & 0.712569\end{array}$


Coordinates of $[3]^{6-} \cdot 12 \mathrm{H}_{2} \mathrm{O}$ in water

\begin{tabular}{|c|c|c|c|}
\hline Atom & $X$ & Y & Z \\
\hline $\mathrm{C}$ & 0.000000 & 0.000000 & 0.000000 \\
\hline $\mathrm{C}$ & 0.000000 & 0.000000 & 1.435758 \\
\hline $\mathrm{C}$ & 1.216474 & 0.000000 & -0.702199 \\
\hline $\mathrm{C}$ & 1.215910 & -0.000723 & 2.138541 \\
\hline $\mathrm{C}$ & 2.459763 & 0.000762 & 1.420967 \\
\hline $\mathrm{C}$ & 2.459781 & 0.001162 & 0.016221 \\
\hline $\mathrm{C}$ & 0.061345 & 0.002076 & 4.143806 \\
\hline $\mathrm{C}$ & -1.162240 & 0.002535 & 3.437240 \\
\hline $\mathrm{H}$ & -2.102854 & 0.004960 & 3.975930 \\
\hline $\mathrm{H}$ & 0.064801 & 0.004133 & 5.227348 \\
\hline $\mathrm{C}$ & -1.160193 & 0.001688 & -2.002021 \\
\hline $\mathrm{C}$ & 0.063408 & 0.001458 & -2.708305 \\
\hline $\mathrm{H}$ & -2.100355 & 0.002860 & -2.541125 \\
\hline $\mathrm{H}$ & 0.067220 & 0.002052 & -3.791870 \\
\hline $\mathrm{C}$ & 4.773618 & 0.006326 & 1.424686 \\
\hline $\mathrm{H}$ & 5.710001 & 0.008566 & 1.969732 \\
\hline $\mathrm{C}$ & 4.773766 & 0.006985 & 0.012136 \\
\hline $\mathrm{H}$ & 5.710401 & 0.008579 & -0.532870 \\
\hline $\mathrm{N}$ & -1.214849 & 0.000629 & 2.079466 \\
\hline $\mathrm{N}$ & -1.214332 & 0.000492 & -0.644862 \\
\hline $\mathrm{N}$ & 1.265453 & 0.000239 & -2.076163 \\
\hline $\mathrm{N}$ & 3.625194 & 0.003267 & -0.713170 \\
\hline $\mathrm{N}$ & 3.625233 & 0.001821 & 2.150628 \\
\hline $\mathrm{N}$ & 1.263857 & -0.001884 & 3.512336 \\
\hline $\mathrm{Ru}$ & 3.265803 & -0.000950 & -2.814304 \\
\hline $\mathrm{Ru}$ & 3.262532 & -0.001758 & 4.253095 \\
\hline $\mathrm{Ru}$ & -2.853874 & 0.000469 & 0.719383 \\
\hline $\mathrm{C}$ & 3.282850 & -2.048131 & -2.848921 \\
\hline $\mathrm{N}$ & 3.286711 & -3.240263 & -2.866514 \\
\hline $\mathrm{C}$ & 5.194955 & -0.002935 & -3.303102 \\
\hline $\mathrm{N}$ & 6.356876 & -0.005237 & -3.575880 \\
\hline $\mathrm{C}$ & 2.724396 & -0.003292 & -4.729451 \\
\hline $\mathrm{N}$ & 2.379806 & -0.005235 & -5.872124 \\
\hline $\mathrm{C}$ & 3.284358 & 2.045954 & -2.855114 \\
\hline $\mathrm{N}$ & 3.294599 & 3.237980 & -2.877094 \\
\hline $\mathrm{C}$ & 5.190060 & 0.002075 & 4.750483 \\
\hline $\mathrm{N}$ & 6.350874 & 0.004855 & 5.027974 \\
\hline $\mathrm{C}$ & 2.715774 & -0.003356 & 6.166204 \\
\hline $\mathrm{N}$ & 2.366830 & -0.004092 & 7.307577 \\
\hline $\mathrm{C}$ & 3.286016 & -2.048942 & 4.288064 \\
\hline $\mathrm{N}$ & 3.295597 & -3.241135 & 4.304607 \\
\hline $\mathrm{C}$ & 3.279566 & 2.045028 & 4.290316 \\
\hline $\mathrm{N}$ & 3.291143 & 3.237140 & 4.305542 \\
\hline $\mathrm{C}$ & -2.895548 & 2.047435 & 0.715663 \\
\hline $\mathrm{N}$ & -2.914357 & 3.239541 & 0.710004 \\
\hline $\mathrm{C}$ & -4.245965 & -0.000153 & -0.704647 \\
\hline $\mathrm{N}$ & -5.064258 & 0.000147 & -1.573392 \\
\hline $\mathrm{C}$ & -4.235745 & 0.001148 & 2.150156 \\
\hline $\mathrm{N}$ & -5.049238 & 0.001996 & 3.023306 \\
\hline $\mathrm{C}$ & -2.896835 & -2.046345 & 0.716503 \\
\hline $\mathrm{N}$ & -2.919967 & -3.238400 & 0.715258 \\
\hline $\mathrm{H}$ & 3.312630 & 4.973271 & -2.915160 \\
\hline $\mathrm{O}$ & 3.297199 & 5.972993 & -2.886657 \\
\hline $\mathrm{H}$ & 2.879831 & 6.278625 & -2.052397 \\
\hline $\mathrm{H}$ & 3.322315 & 4.972067 & 4.350360 \\
\hline $\mathrm{O}$ & 3.311714 & 5.972094 & 4.331028 \\
\hline $\mathrm{H}$ & 2.819932 & 6.287813 & 3.542327 \\
\hline
\end{tabular}




$\begin{array}{crrr}\mathrm{H} & -2.952317 & 4.974866 & 0.701042 \\ \mathrm{O} & -2.918158 & 5.974492 & 0.699771 \\ \mathrm{H} & -1.987960 & 6.278717 & 0.775810 \\ \mathrm{H} & -2.964942 & -4.973684 & 0.713885 \\ \mathrm{O} & -2.934604 & -5.973454 & 0.710902 \\ \mathrm{H} & -2.003895 & -6.280932 & 0.657534 \\ \mathrm{H} & 3.310483 & -4.976756 & 4.330360 \\ \mathrm{O} & 3.291163 & -5.976187 & 4.295023 \\ \mathrm{H} & 2.838279 & -6.274429 & 3.476769 \\ \mathrm{H} & 3.300402 & -4.975135 & -2.909739 \\ \mathrm{O} & 3.285498 & -5.975007 & -2.884930 \\ \mathrm{H} & 2.907634 & -6.284213 & -2.033341 \\ \mathrm{H} & 1.893772 & -0.008428 & -7.533199 \\ \mathrm{O} & 1.559769 & -0.008814 & -8.476872 \\ \mathrm{H} & 0.578727 & 0.022710 & -8.485958 \\ \mathrm{H} & 8.038727 & -0.008949 & -3.980631 \\ \mathrm{O} & 9.023409 & -0.008956 & -4.160777 \\ \mathrm{H} & 9.519667 & 0.033269 & -3.314920 \\ \mathrm{H} & 8.033578 & 0.008872 & 5.430149 \\ \mathrm{O} & 9.018743 & 0.011122 & 5.607857 \\ \mathrm{H} & 9.512995 & 0.007976 & 4.759777 \\ \mathrm{H} & 1.872591 & -0.005079 & 8.966239 \\ \mathrm{O} & 1.533780 & -0.006027 & 9.908201 \\ \mathrm{H} & 0.552257 & -0.016084 & 9.912071 \\ \mathrm{H} & -6.235422 & 0.003312 & 4.282796 \\ \mathrm{O} & -6.880822 & 0.003574 & 5.047883 \\ \mathrm{H} & -6.392667 & -0.006123 & 5.899434 \\ \mathrm{H} & -6.262452 & 0.001185 & -2.821596 \\ \mathrm{O} & -6.915848 & -0.000198 & -3.579944 \\ \mathrm{H} & -6.437391 & -0.045656 & -4.435812\end{array}$


Coordinates of $[2]^{4-} \cdot 8 \mathrm{H}_{2} \mathrm{O}$ in water

$\begin{array}{llll}\text { Atom } & \mathrm{X} & \mathrm{Y} & \mathrm{Z}\end{array}$

$\begin{array}{llll}\text { C } & -0.000515 & 0.005220 & 0.003611\end{array}$

$\begin{array}{llll}\text { C } & -0.000045 & 0.003692 & 1.438458\end{array}$

$\begin{array}{llll}\text { C } & 1.214143 & 0.003589 & -0.693298\end{array}$

$\begin{array}{llll}\text { C } & 1.208871 & 0.000427 & 2.182732\end{array}$

$\begin{array}{llll}\text { C } & 2.484438 & -0.002760 & 1.451007\end{array}$

$\begin{array}{llll}\text { C } & 2.452561 & -0.000592 & 0.031648\end{array}$

$\begin{array}{llll}\text { C } & -0.029425 & 0.002442 & 4.136879\end{array}$

C $\quad-1.241159 \quad 0.005644 \quad 3.404044$

H $\quad-2.199969 \quad 0.007468 \quad 3.909129$

$\begin{array}{llll}\mathrm{H} & -0.057367 & 0.002054 & 5.225067\end{array}$

$\begin{array}{llll}\text { C } & -1.158288 & 0.010818 & -2.004135\end{array}$

$\begin{array}{llll}\text { C } & 0.065141 & 0.009285 & -2.706085\end{array}$

$\mathrm{H} \quad-2.098001 \quad 0.013789 \quad-2.543348$

H $\quad 0.074040 \quad 0.010972 \quad-3.789428$

$\begin{array}{llll}\text { C } & 4.796519 & -0.009231 & 1.368811\end{array}$

H $\quad 5.749808 \quad-0.012914 \quad 1.894260$

$\begin{array}{llll}\text { C } & 4.775969 & -0.006895 & -0.047057\end{array}$

H $\quad 5.695979 \quad-0.008467 \quad-0.619758$

$\begin{array}{llll}\mathrm{N} & -1.234975 & 0.006164 & 2.052584\end{array}$

$\begin{array}{llll}\mathrm{N} & -1.212747 & 0.008877 & -0.647322\end{array}$

$\begin{array}{llll}\mathrm{N} & 1.263921 & 0.005717 & -2.068351\end{array}$

N $3.606239 \quad-0.002406 \quad-0.724047$

$\begin{array}{llll}\mathrm{N} & 3.675045 & -0.007037 & 2.120744\end{array}$

N $\quad \begin{array}{llll}1.185797 & -0.000374 & 3.548513\end{array}$

$\begin{array}{llll}\mathrm{Ru} & 3.244037 & 0.002971 & -2.822793\end{array}$

$\begin{array}{llll}\mathrm{Ru} & -2.863693 & 0.011508 & 0.680890\end{array}$

$\begin{array}{llll}\text { C } & 3.262553 & -2.044006 & -2.856119\end{array}$

N $3.273838 \quad-3.236316 \quad-2.868589$

$\begin{array}{llll}\text { C } & 5.169887 & 0.000312 & -3.333835\end{array}$

$\begin{array}{llll}\mathrm{N} & 6.327880 & -0.001364 & -3.623608\end{array}$

$\begin{array}{llll}\text { C } & 2.693534 & 0.007652 & -4.735756\end{array}$

N $\quad 2.338500 \quad 0.009854 \quad-5.875466$

C $3.271847 \quad 2.049755 \quad-2.848620$

N $\quad 3.289713 \quad 3.242004 \quad-2.860528$

$\begin{array}{llll}\text { C } & -2.894194 & 2.058724 & 0.689078\end{array}$

$\begin{array}{llll}\mathrm{N} & -2.907880 & 3.251054 & 0.695515\end{array}$

$\begin{array}{llll}\text { C } & -4.239505 & 0.023318 & -0.758228\end{array}$

$\begin{array}{llll}\mathrm{N} & -5.046673 & 0.032921 & -1.637567\end{array}$

$\begin{array}{llll}\text { C } & -4.274591 & 0.013488 & 2.087907\end{array}$

$\begin{array}{llll}\mathrm{N} & -5.104746 & 0.015566 & 2.945684\end{array}$

$\begin{array}{llll}\text { C } & -2.910085 & -2.035007 & 0.680571\end{array}$

$\begin{array}{llll}\mathrm{N} & -2.936685 & -3.227154 & 0.684150\end{array}$

$\mathrm{H} \quad 3.325385 \quad 4.977370 \quad-2.881535$

O $\quad 3.306540 \quad 5.977104 \quad-2.850521$

H $\quad 2.627491 \quad 6.280354 \quad-2.210202$

H $\quad-2.946216 \quad 4.986390 \quad 0.713553$

$\begin{array}{llll}\text { O } & -2.912299 & 5.986179 & 0.714085\end{array}$

$\mathrm{H} \quad-2.004703 \quad 6.291692 \quad 0.499562$

H $\quad-2.989669-4.962072 \quad 0.684306$

$\begin{array}{llll}\mathrm{O} & -2.964425 & -5.962035 & 0.669854\end{array}$

H $\quad-2.079572 \quad-6.271460 \quad 0.379318$

H $3.301822 \quad-4.971351 \quad-2.908915$

O $3.281473 \quad-5.971340 \quad-2.889234$

H $\quad 2.646150 \quad-6.281066 \quad-2.208445$

H $\quad 1.844542 \quad 0.013292 \quad-7.534756$

$\begin{array}{lllll}\text { O } & 1.505695 & 0.018002 & -8.476804\end{array}$ 
$\begin{array}{llll}\mathrm{H} & 0.525742 & 0.069788 & -8.480981\end{array}$

H $\quad 8.006432 \quad-0.001221 \quad-4.043025$

$\begin{array}{lllll}\text { O } & 8.990428 & 0.001114 & -4.227806\end{array}$

H $\quad 9.490472 \quad 0.039425 \quad-3.384343$

$\begin{array}{llll}\mathrm{H} & -6.309937 & 0.019391 & 4.187416\end{array}$

$\begin{array}{llll}\mathrm{O} & -6.964319 & 0.022937 & 4.945121\end{array}$

H $\quad-6.486434 \quad 0.050147 \quad 5.801758$

H $\quad-6.234734 \quad 0.051190 \quad-2.895499$

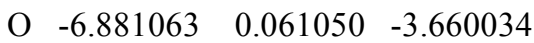

H $\quad-6.394509 \quad 0.036148 \quad-4.511881$ 
Coordinates of $[\mathbf{1}]^{2-} \cdot 4 \mathrm{H}_{2} \mathrm{O}$ in water

$\begin{array}{cccc}\text { Atom } & \mathrm{X} & \mathrm{Y} & \mathrm{Z} \\ \mathrm{C} & -0.028276 & 0.023048 & 0.012757 \\ \mathrm{C} & -0.021666 & 0.007964 & 1.479599 \\ \mathrm{C} & 1.213093 & 0.013257 & -0.665358 \\ \mathrm{C} & 1.218913 & -0.014542 & 2.194663 \\ \mathrm{C} & 2.491622 & -0.023455 & 1.465064 \\ \mathrm{C} & 2.456090 & -0.009230 & 0.051016 \\ \mathrm{C} & 0.054984 & -0.020593 & 4.202980 \\ \mathrm{C} & -1.176026 & 0.002258 & 3.493435 \\ \mathrm{H} & -2.125704 & 0.008933 & 4.025200 \\ \mathrm{H} & 0.070897 & -0.031785 & 5.291285 \\ \mathrm{C} & -1.118009 & 0.057192 & -2.028417 \\ \mathrm{C} & 0.116603 & 0.047074 & -2.718726 \\ \mathrm{H} & -2.050093 & 0.074937 & -2.590106 \\ \mathrm{H} & 0.154169 & 0.056710 & -3.801900 \\ \mathrm{C} & 4.804069 & -0.051367 & 1.384535 \\ \mathrm{H} & 5.757413 & -0.068603 & 1.909348 \\ \mathrm{C} & 4.782516 & -0.037957 & -0.029692 \\ \mathrm{H} & 5.701080 & -0.044412 & -0.604867 \\ \mathrm{~N} & -1.214714 & 0.016434 & 2.149387 \\ \mathrm{~N} & -1.205378 & 0.045175 & -0.681748 \\ \mathrm{~N} & 1.287577 & 0.024814 & -2.043857 \\ \mathrm{~N} & 3.611433 & -0.016645 & -0.704735 \\ \mathrm{~N} & 3.682662 & -0.044333 & 2.135499 \\ \mathrm{~N} & 1.237355 & -0.028717 & 3.562637 \\ \mathrm{Ru} & 3.268127 & 0.004463 & -2.793735 \\ \mathrm{C} & 3.255593 & -2.041872 & -2.840813 \\ \mathrm{~N} & 3.242509 & -3.234172 & -2.857998 \\ \mathrm{C} & 5.196822 & -0.015214 & -3.295315 \\ \mathrm{~N} & 6.356934 & -0.032899 & -3.576213 \\ \mathrm{O} & 8.028639 & -0.062541 & -3.989358 \\ \mathrm{H} & 9.010689 & -0.058663 & -4.188890 \\ \mathrm{~N} & 2.731196 & 0.027018 & -4.713117 \\ \mathrm{C} & 3.381874 & 0.052843 & -5.854298 \\ \mathrm{~N} & 3.329835 & 2.050585 & -2.814392 \\ \mathrm{H} & 3.363004 & 3.242918 & -2.819945 \\ \mathrm{O} & 3.344629 & 5.972944 & -2.800882 \\ \mathrm{H} & 2.624168 & 6.278424 & -2.209080 \\ \mathrm{H} & 3.239215 & -4.963255 & -2.902409 \\ \mathrm{O} & 3.206548 & -5.963767 & -2.889868 \\ \mathrm{H} & 2.592662 & -6.272246 & -2.189438 \\ \mathrm{H} & 1.894122 & 0.105783 & -7.506221 \\ \mathrm{H} & 0.967313 & -0.641580 & -8.612871 \\ \mathrm{H} & & -3.525180\end{array}$


Table S3. Analytical and IR spectroscopic data for the new complexes Complex

Elemental Analysis

$(\% \mathrm{C}, \% \mathrm{H}, \% \mathrm{~N})^{a}$

Principal IR spectral peaks, $\mathrm{cm}^{-1 b}$

Derivatives of $\left[\mathrm{Ru}(\mathrm{CN})_{4}(\mathrm{HAT})\right]^{2-}$

\begin{tabular}{|c|c|c|}
\hline $\mathrm{Na}_{2}\left[\left\{\mathrm{Ru}(\mathrm{CN})_{4}\right\}(\mathrm{HAT})\right] \bullet 4 \mathrm{H}_{2} \mathrm{O}$ & $33.9(34.4), 2.3(2.5), 25.0(25.1)$ & $\begin{array}{l}3431,2105,2073,2062,1630 \text {, } \\
1384\end{array}$ \\
\hline$(\mathrm{PPN})_{2}\left[\left\{\mathrm{Ru}(\mathrm{CN})_{4}\right\}(\mathrm{HAT})\right] \cdot 5 \mathrm{H}_{2} \mathrm{O}^{e}$ & $65.6(65.8), 4.4(4.7), 10.3(10.5)$ & $\begin{array}{l}3435,3056,2099,2071,1639, \\
1438,1290,1266,1115,724, \\
693,534\end{array}$ \\
\hline $\begin{array}{l}{\left[\left\{\mathrm{Ru}(\mathrm{CN})_{4}\right\}(\mathrm{HAT})\right]_{1.5}\left[\mathrm{Nd}\left(\mathrm{H}_{2} \mathrm{O}\right)_{5}\right]} \\
\cdot 6.5 \mathrm{H}_{2} \mathrm{O}^{c}[\mathrm{Nd}(\mathbf{1})]\end{array}$ & $28.6(28.5), 2.9(3.2), 20.6(20.8)$ & $\begin{array}{l}3435,2113,2101,2083,2060, \\
1632,1384\end{array}$ \\
\hline $\begin{array}{l}{\left[\left\{\mathrm{Ru}(\mathrm{CN})_{4}\right\}(\mathrm{HAT})\right]_{1.5}\left[\mathrm{Sm}\left(\mathrm{H}_{2} \mathrm{O}\right)_{5}\right]} \\
\cdot 5.5 \mathrm{H}_{2} \mathrm{O}[\mathrm{Sm}(\mathbf{1})]\end{array}$ & $28.2(28.7), 2.8(3.0), 20.7(21.0)$ & $\begin{array}{l}3422,1114,2102,2182,2061, \\
1628,1385\end{array}$ \\
\hline $\begin{array}{l}{\left[\left\{\mathrm{Ru}(\mathrm{CN})_{4}\right\}(\mathrm{HAT})\right]_{1.5}\left[\mathrm{Eu}\left(\mathrm{H}_{2} \mathrm{O}\right)_{5}\right]} \\
\cdot 8 \mathrm{H}_{2} \mathrm{O}[\mathrm{Eu}(\mathbf{1})]\end{array}$ & $27.3(27.5), 2.7(3.3), 20.0(20.1)$ & $\begin{array}{l}3436,2113,2102,2079,2061, \\
1634,1384\end{array}$ \\
\hline $\begin{array}{l}{\left[\left\{\mathrm{Ru}(\mathrm{CN})_{4}\right\}(\mathrm{HAT})\right]_{1.5}\left[\mathrm{Gd}\left(\mathrm{H}_{2} \mathrm{O}\right)_{5}\right]} \\
\cdot 6.5 \mathrm{H}_{2} \mathrm{O}^{d}[\mathrm{Gd}(\mathbf{1})]\end{array}$ & $28.1(28.1), 2.9(3.1), 20.5(20.5)$ & $\begin{array}{l}3395,2114,2103,2079,2061, \\
1629,1385\end{array}$ \\
\hline $\begin{array}{l}{\left[\left\{\mathrm{Ru}(\mathrm{CN})_{4}\right\}(\mathrm{HAT})\right]\left[\mathrm{Yb}\left(\mathrm{H}_{2} \mathrm{O}\right)_{5}\right]} \\
{\left[\mathrm{Yb}\left(\mathrm{NO}_{3}\right)_{2}\left(\mathrm{H}_{2} \mathrm{O}\right)_{3}\right]\left(\mathrm{NO}_{3}\right)_{2} \bullet 6.5 \mathrm{H}_{2} \mathrm{O}} \\
{[\mathrm{Yb}(\mathbf{1})]}\end{array}$ & $14.5(14.6), 2.5(2.8), 15.0(14.9)$ & $3412,2139,2094,1631,1384$ \\
\hline \multicolumn{3}{|c|}{ Derivatives of $\left[\left\{\mathrm{Ru}(\mathrm{CN})_{4}\right\}_{2}\left(\mu^{2}-\mathrm{HAT}\right)\right]^{4-}$} \\
\hline $\mathrm{Na}_{4}\left[\left\{\mathrm{Ru}(\mathrm{CN})_{4}\right\}_{2}\left(\mu^{2}-\mathrm{HAT}\right)\right] \bullet 8 \mathrm{H}_{2} \mathrm{O}^{e}$ & $27.5(27.2), 2.0(2.5), 22.6(22.2)$ & $\begin{array}{l}3436,2100,2068,2057,1632, \\
1388\end{array}$ \\
\hline $\begin{array}{l}(\mathrm{PPN})_{4}\left[\left\{\mathrm{Ru}(\mathrm{CN})_{4}\right\}_{2}\left(\mu^{2}-\mathrm{HAT}\right)\right] \\
\cdot 14 \mathrm{H}_{2} \mathrm{O}^{e}\end{array}$ & $64.6(64.6), 4.8(5.1), 8.2(8.3)$ & $\begin{array}{l}3435.3056,2097.2069,1637, \\
1438,1263,1115,724,694, \\
550,533\end{array}$ \\
\hline $\begin{array}{l}{\left[\left\{\mathrm{Ru}(\mathrm{CN})_{4}\right\}_{2}\left(\mu^{2}-\mathrm{HAT}\right)\right]} \\
{\left[\mathrm{Nd}\left(\mathrm{NO}_{3}\right)\left(\mathrm{H}_{2} \mathrm{O}\right)_{5}\right]_{2} \bullet 4 \mathrm{H}_{2} \mathrm{O}^{e}[\mathrm{Nd}(2)]}\end{array}$ & $18.2(18.4), 2.2(2.6), 16.9(17.2)$ & $\begin{array}{l}3407,2114,2076,2066,1614, \\
1385\end{array}$ \\
\hline $\begin{array}{l}{\left[\left\{\mathrm{Ru}(\mathrm{CN})_{4}\right\}_{2}\left(\mu^{2}-\mathrm{HAT}\right)\right]} \\
{\left[\mathrm{Gd}\left(\mathrm{NO}_{3}\right)\left(\mathrm{H}_{2} \mathrm{O}\right)_{5}\right]_{2} \bullet 4 \mathrm{H}_{2} \mathrm{O}^{e}[\mathrm{Gd}(2)]}\end{array}$ & $17.9(18.2), 2.0(2.4), 16.8(17.0)$ & $\begin{array}{l}3430,2123,2088,2064,1614, \\
1385\end{array}$ \\
\hline $\begin{array}{l}{\left[\left\{\mathrm{Ru}(\mathrm{CN})_{4}\right\}_{2}\left(\mu^{2}-\mathrm{HAT}\right)\right]_{2}} \\
{\left[\mathrm{Yb}\left(\mathrm{NO}_{3}\right)\left(\mathrm{H}_{2} \mathrm{O}\right)_{2}\right]\left[\mathrm{Yb}\left(\mathrm{H}_{2} \mathrm{O}\right)_{4}\right]}\end{array}$ & 19.1 (19.5), $2.7(3.1), 16.3$ (16.5) & $\begin{array}{l}3410,2122,2081,2064,1617, \\
1385\end{array}$ \\
\hline
\end{tabular}

$\left[\mathrm{Yb}\left(\mathrm{H}_{2} \mathrm{O}\right)_{6}\right] \cdot 20 \mathrm{H}_{2} \mathrm{O}[\mathrm{Yb}(2)]$

Derivatives of $\left[\left\{\mathrm{Ru}(\mathrm{CN})_{4}\right\}_{3}\left(\mu^{3}-\mathrm{HAT}\right)\right]^{6-}$

\begin{tabular}{|c|c|c|}
\hline $\mathrm{Na}_{6}\left[\left\{\mathrm{Ru}(\mathrm{CN})_{4}\right\}_{3}\left(\mu^{3}-\mathrm{HAT}\right)\right] \cdot 12 \mathrm{H}_{2} \mathrm{O}^{e}$ & 23.6 (23.9), $2.4(2.5), 20.5(20.9)$ & $3436,2102,2070,1630,1390$ \\
\hline $\begin{array}{l}(\mathrm{PPN})_{6}\left[\left\{\mathrm{Ru}(\mathrm{CN})_{4}\right\}_{3}\left(\mu^{3}-\mathrm{HAT}\right)\right] \\
\cdot 10 \mathrm{H}_{2} \mathrm{O}^{e}\end{array}$ & $67.5(67.6), 4.9(4.9), 8.1(7.9)$ & $\begin{array}{l}3434,2093,2068,1635,1437 \\
1264,1114,723,693,548,534\end{array}$ \\
\hline $\begin{array}{l}{\left[\mathrm{Nd}\left(\mathrm{H}_{2} \mathrm{O}\right)_{5}\right]_{2}\left[\left\{\mathrm{Ru}(\mathrm{CN})_{4}\right\}_{3}\left(\mu^{3}-\mathrm{HAT}\right)\right]} \\
\cdot 13 \mathrm{H}_{2} \mathrm{O}[\mathrm{Nd}(\mathbf{3})]\end{array}$ & $18.2(18.6), 2.9(3.4), 15.8(16.2)$ & $\begin{array}{l}3392,2121,2085,2070,1620, \\
1385\end{array}$ \\
\hline $\begin{array}{l}{\left[\mathrm{Gd}\left(\mathrm{H}_{2} \mathrm{O}\right)_{5}\right]_{2}\left[\left\{\mathrm{Ru}(\mathrm{CN})_{4}\right\}_{3}\left(\mu^{3}-\mathrm{HAT}\right)\right]} \\
\cdot 13 \mathrm{H}_{2} \mathrm{O}^{e}[\mathrm{Gd}(\mathbf{3})]\end{array}$ & $18.0(18.2), 3.3(3.3), 15.5(15.9)$ & $\begin{array}{l}3380,2126,2091,2068,1630, \\
1384\end{array}$ \\
\hline $\begin{array}{l}{\left[\mathrm{Yb}\left(\mathrm{H}_{2} \mathrm{O}\right)_{4}\right]_{2}\left[\left\{\mathrm{Ru}(\mathrm{CN})_{4}\right\}_{3}\left(\mu^{3}-\mathrm{HAT}\right)\right]} \\
\cdot 13 \mathrm{H}_{2} \mathrm{O}[\mathrm{Yb}(\mathbf{3})]\end{array}$ & $17.9(18.3), 2.9(3.1), 15.7(16.0)$ & $\begin{array}{l}3367,2134,2098,2068,1610, \\
1384\end{array}$ \\
\hline \multicolumn{3}{|c|}{ a Calculated figures are in parentheses. } \\
\hline \multirow{2}{*}{\multicolumn{3}{|c|}{$\begin{array}{l}b \text { Recorded on the pure crystalline solids using an ATR accessory. The set of peaks between } 2000 \\
\text { and } 2150 \mathrm{~cm}^{-1} \text { arises from cyanide vibrations. Only the most intense IR peaks are listed. } \\
\text { The crystal structure has } 9 \mathrm{H}_{2} \mathrm{O} \text {. }\end{array}$}} \\
\hline & & \\
\hline \multicolumn{3}{|l|}{$d \quad$ The crystal structure has $8 \mathrm{H}_{2} \mathrm{O}$. } \\
\hline
\end{tabular}


Table S4. Crystal parameters, data collection and refinement details for the structures in this paper.

\begin{tabular}{|l|l|l|l|}
\hline Complex & $\mathrm{Na}_{2}[\mathbf{1}] \cdot 4 \mathrm{H}_{2} \mathrm{O}$ & {$\left[\left\{\mathrm{Nd}\left(\mathrm{H}_{2} \mathrm{O}\right)_{5}\right\}_{2}\{\mathbf{1}\}_{3} \bullet 18 \mathrm{H}_{2} \mathrm{O}\right]_{\infty}$} & $\begin{array}{l}{\left[\left\{\mathrm{Sm}\left(\mathrm{H}_{2} \mathrm{O}\right)_{5}\right\}_{2}\{\mathbf{1}\}_{3} \bullet\right.} \\
\left.11 \mathrm{H}_{2} \mathrm{O}\right]_{\infty}\end{array}$ \\
\hline Formula & $\mathrm{C}_{16} \mathrm{H}_{14} \mathrm{~N}_{10} \mathrm{Na}_{2} \mathrm{O}_{4} \mathrm{Ru}$ & $\mathrm{C}_{48} \mathrm{H}_{74} \mathrm{~N}_{30} \mathrm{Nd}_{2} \mathrm{O}_{28} \mathrm{Ru}_{3}$ & $\mathrm{C}_{48} \mathrm{H}_{60} \mathrm{~N}_{30} \mathrm{O}_{21} \mathrm{Sm}_{2} \mathrm{Ru}_{3}$ \\
\hline Molecular weight & 557.42 & 2111.06 & 1997.17 \\
\hline$T, \mathrm{~K}$ & $150(2)$ & $150(2)$ & $150(2)$ \\
\hline Crystal system & Monoclinic & Monoclinic & Monoclinic \\
\hline Space group & $P 2(1) / \mathrm{n}$ & $C 2 / \mathrm{c}$ & $C 2 / \mathrm{c}$ \\
\hline$a, \AA$ & $9.862(3)$ & $33.916(7)$ & $33.057(10)$ \\
\hline$b, \AA$ & $16.668(5)$ & $13.304(3)$ & $13.055(4)$ \\
\hline$c, \AA$ & $13.171(4)$ & $19.723(4)$ & $19.390(6)$ \\
\hline$\alpha,{ }^{\circ}$ & 90 & 90 & 90 \\
\hline$\beta,{ }^{\circ}$ & $107.354(5)$ & $124.70(3)$ & $124.669(4)$ \\
\hline$\gamma,{ }^{\circ}$ & 90 & 90 & 90 \\
\hline$V, \AA^{3}$ & $2066.5(11)$ & $7316(3)$ & $6882(3)$ \\
\hline $\mathrm{Z}$ & 4 & 4 & 4 \\
\hline$\rho, \mathrm{g} \mathrm{cm}^{-3}$ & 1.792 & 1.917 & 1.927 \\
\hline Crystal size, $\mathrm{mm}^{3}$ & $0.40 \times 0.24 \times 0.10$ & $0.15 \times 0.12 \times 0.07$ & $0.19 \times 0.18 \times 0.07$ \\
\hline$\mu, \mathrm{mm}^{-1}$ & 0.850 & 2.100 & 2.417 \\
\hline $\begin{array}{l}\text { Data, restraints, } \\
\text { parameters }\end{array}$ & $4647,12,322$ & $6437,83,491$ & $6069,0,465$ \\
\hline Final $R 1, w R 2$ & $0.0317,0.0697$ & $0.0561,0.1462$ & $0.0746,0.2188$ \\
\hline
\end{tabular}

\begin{tabular}{|l|l|l|l|}
\hline Complex & $\begin{array}{l}{\left[\left\{\mathrm{Eu}\left(\mathrm{H}_{2} \mathrm{O}\right)_{5}\right\}_{2}\{\mathbf{1}\}_{3} 3^{\bullet}\right.} \\
\left.16 \mathrm{H}_{2} \mathrm{O}\right]_{\infty}\end{array}$ & $\begin{array}{l}{\left[\left\{\mathrm{Gd}\left(\mathrm{H}_{2} \mathrm{O}\right)_{5}\right\}_{2}\{\mathbf{1}\}_{3} 3^{\bullet}\right.} \\
\left.16 \mathrm{H}_{2} \mathrm{O}\right]_{\infty}\end{array}$ & $\begin{array}{l}{\left[\left\{\mathrm{Yb}\left(\mathrm{H}_{2} \mathrm{O}\right)_{3}\left(\mathrm{NO}_{3}\right)_{2}\right\}\{\mathrm{Yb}\right.} \\
\left.\left.\left(\mathrm{H}_{2} \mathrm{O}\right)_{6}\right\}\{\mathbf{1}\}\left(\mathrm{NO}_{3}\right)_{2}\left(\mathrm{H}_{2} \mathrm{O}\right)_{6.5}\right]_{\infty}\end{array}$ \\
\hline Formula & $\mathrm{C}_{48} \mathrm{H}_{70} \mathrm{Eu}_{2} \mathrm{~N}_{30} \mathrm{O}_{26} \mathrm{Ru}_{3}$ & $\mathrm{C}_{48} \mathrm{H}_{70} \mathrm{Gd}_{2} \mathrm{~N}_{30} \mathrm{O}_{26} \mathrm{Ru}_{3}$ & $\mathrm{C}_{16} \mathrm{H}_{37} \mathrm{~N}_{14} \mathrm{O}_{27.5} \mathrm{RuYb}_{2}$ \\
\hline Molecular weight & 2090.47 & 2101.05 & 1312.75 \\
\hline$T, \mathrm{~K}$ & $150(2)$ & $150(2)$ & $150(2)$ \\
\hline Crystal system & Monoclinic & Monoclinic & Triclinic \\
\hline Space group & $C 2 / \mathrm{c}$ & $C 2 / \mathrm{c}$ & $P-1$ \\
\hline$a, \AA$ & $33.824(6)$ & $33.778(14)$ & $10.680(2)$ \\
\hline$b, \AA$ & $13.260(2)$ & $13.233(6)$ & $14.001(3)$ \\
\hline$c, \AA$ & $19.672(4)$ & $19.647(8)$ & $14.272(3)$ \\
\hline$\alpha,{ }^{\circ}$ & 90 & 90 & $76.75(3)$ \\
\hline$\beta,{ }^{\circ}$ & $124.627(3)$ & $124.616(6)$ & $79.50(3)$ \\
\hline$\gamma,{ }^{\circ}$ & 90 & 90 & $76.25(3)$ \\
\hline$V, \AA^{3}$ & $7260(2)$ & $7228(5)$ & $1999.2(7)$ \\
\hline $\mathrm{Z}$ & 4 & 1.931 & 2 \\
\hline$\rho, \mathrm{g} \mathrm{cm}^{-3}$ & 1.913 & $0.49 \times 0.49 \times 0.32$ & 2.181 \\
\hline Crystal size, $\mathrm{mm}^{3}$ & $0.30 \times 0.09 \times 0.09$ & 2.521 & $0.14 \times 0.09 \times 0.03$ \\
\hline$\mu, m^{-1}$ & 2.410 & $6351,0,487$ & $7007,3,542$ \\
\hline $\begin{array}{l}\text { Data, restraints, } \\
\text { parameters }\end{array}$ & $6339,92,491$ & & \\
\hline Final $R 1, w R 2$ & $0.0706,0.1934$ & $0.0546,0.1499$ & $0.0292,0.0757$ \\
\hline
\end{tabular}

...continued on next page... 
...Table S4 (continued)

\begin{tabular}{|c|c|c|c|}
\hline Complex & $\begin{array}{l}{\left[\left\{\mathrm{Yb}\left(\mathrm{H}_{2} \mathrm{O}\right)_{4}\right\}\left\{\mathrm{Yb}\left(\mathrm{H}_{2} \mathrm{O}\right)_{6}\right\}\right.} \\
\left\{\mathrm{Yb}\left(\mathrm{H}_{2} \mathrm{O}\right)_{2}\left(\mathrm{NO}_{3}\right)\right\}\{2\}_{2} \\
\left.\cdot 20\left(\mathrm{H}_{2} \mathrm{O}\right)\right]_{\infty}\end{array}$ & $\begin{array}{l}{\left[\{\mathbf{3}\}\left\{\mathrm{Nd}\left(\mathrm{H}_{2} \mathrm{O}\right)_{5}\right\}_{2}\right.} \\
\left.\left(\mathrm{H}_{2} \mathrm{O}\right)_{13}\right]_{\infty}\end{array}$ & $\begin{array}{l}{\left[\{\mathbf{3}\}\left\{\mathrm{Yb}\left(\mathrm{H}_{2} \mathrm{O}\right)_{4}\right\}_{2}\right.} \\
\left.\left(\mathrm{H}_{2} \mathrm{O}\right)_{13}\right]_{\infty}\end{array}$ \\
\hline Formula & $\mathrm{C}_{40} \mathrm{H}_{76} \mathrm{~N}_{29} \mathrm{O}_{35} \mathrm{Ru}_{4} \mathrm{Yb}_{3}$ & $\mathrm{C}_{24} \mathrm{H}_{52} \mathrm{~N}_{18} \mathrm{Nd}_{2} \mathrm{O}_{23} \mathrm{Ru}_{3}$ & $\mathrm{C}_{24} \mathrm{H}_{48} \mathrm{~N}_{18} \mathrm{O}_{21} \mathrm{Ru}_{3} \mathrm{Yb}_{2}$ \\
\hline Molecular weight & 2446.70 & 1552.53 & 1574.09 \\
\hline$T, \mathrm{~K}$ & $100(2)$ & $150(2)$ & $150(2)$ \\
\hline Crystal system & Triclinic & Monoclinic & Monoclinic \\
\hline Space group & $P-1$ & $P 2_{1} / \mathrm{m}$ & $P 2_{1} / \mathrm{m}$ \\
\hline$a, \AA$ & $11.4801(5)$ & $8.7731(7)$ & $8.6800(6)$ \\
\hline$b, \AA$ & $15.8855(6)$ & $25.689(2)$ & $25.0903(15)$ \\
\hline$c, \AA$ & $22.8864(10)$ & $11.0330(9)$ & $11.0863(7)$ \\
\hline$\alpha,^{\circ}$ & $97.127(2)$ & 90 & 90 \\
\hline$\beta,^{\circ}$ & $100.460(2)$ & $101.834(4)$ & $101.550(4)$ \\
\hline$\gamma$, & $103.133(2)$ & 90 & 90 \\
\hline$V, \AA^{3}$ & $3937.0(3)$ & $2433.7(3)$ & $2365.5(3)$ \\
\hline $\mathrm{Z}$ & 2 & 2 & 2 \\
\hline$\rho, \mathrm{g} \mathrm{cm}^{-3}$ & 2.064 & 2.119 & 2.210 \\
\hline Crystal size, $\mathrm{mm}^{3}$ & $0.20 \times 0.10 \times 0.08$ & $0.20 \times 0.15 \times 0.07$ & $0.21 \times 0.10 \times 0.07$ \\
\hline$\mu, \mathrm{mm}^{-1}$ & 4.373 & 3.101 & 4.943 \\
\hline $\begin{array}{l}\text { Data, restraints, } \\
\text { parameters }\end{array}$ & $13812,1,960$ & $5651,7,350$ & $4222,18,352$ \\
\hline Final $R 1, w R 2$ & $0.0584,0.1729$ & $0.0668,0.1239$ & $0.0487,0.1416$ \\
\hline
\end{tabular}

(end of Table S4) 